\title{
Blue stragglers in star clusters and the conventional SSP models
}

\author{
Yu Xin, ${ }^{1}$ Richard de Grijs, ${ }^{2,3}$ Licai Deng $^{3}$ and Pavel Kroupa ${ }^{1}$ \\ ${ }^{1}$ Argelander Institute for Astronomy, University of Bonn, Bonn, Germany \\ ${ }^{2}$ Department of Physics and Astronomy, University of Sheffield, Sheffield, UK \\ ${ }^{3}$ National Astronomical Observatories, Chinese Academy of Sciences, Beijing, China
}

\begin{abstract}
The presence of blue straggler stars (BSs) as secure members of star clusters poses a major challenge to the conventional picture of simple stellar population (SSP) models. The models are based on the stellar evolution theory of single stars, while the major formation mechanisms of BSs are all correlated with stellar interactions. Based on a sufficient working sample including 100 Galactic open clusters, one Galactic globular cluster, and seven Magellanic Cloud star clusters, we discuss the modifications of the properties of broad-band colors and Lick indices of the standard SSP models due to BS populations.
\end{abstract}

Keywords. blue stragglers, galaxies: star clusters

The full poster (in pdf format) is available at http://www.astro.iag.usp.br/ iaus266/Posters/pXin.pdf. 